\title{
Solving Third Order Ordinary Differential Equations Directly Using Hybrid Numerical Models
}

\author{
J. O. Kuboye, O. F. Quadri*, O. R. Elusakin \\ Department of Mathematics, Federal University Oye-Ekiti, Nigeria
}

\begin{abstract}
In this work, numerical methods for solving third order initial value problems of ordinary differential equations are developed. Multi-step collocation is used in deriving the methods, where power series approximate solution is employed as a basis function. Gaussian elimination approach is considered in finding the unknown variables $a_{j}, j=0(1) 8$ in interpolation and collocation equations, which are substituted into the power series to give the continuous implicit schemes. The discrete schemes and its derivatives are derived by evaluating the grid and non-grid points. These schemes are arranged in a matrix form to produce the block methods. The order of the developed methods are found to be six. The numerical results proved the efficiency of the methods over the existing methods.
\end{abstract}

Keywords: Interpolation, collocation, block method, multistep method and third order initial value problem

Article History :

Received: 21 January 2020

Received in revised form: 09 March 2020

Accepted for publication: 16 March 2020

Published: 14 May 2020

(C)2020 Journal of the Nigerian Society of Physical Sciences. All rights reserved. Communicated by: T. Latunde

\section{Introduction}

In time past, to solve third order ordinary differential equation, a reduction method is employed and this involves reducing the differentials to three systems of first order ordinary differential equation, and the numerical approach for first order ordinary differential equation is used to solve the resulting system. This technique has a lot of drawbacks like wastage of time as a result of many functions to be evaluated and the computational effort required. This is extensively discussed in Ref. [1-5]. Advancing in numerical methods, direct method for solving higher order initial value problems without going through the process of reduction was developed by Fatunla [2], Kuboye and Omar

${ }^{*}$ Corresponding author tel. no: +2347068522865

Email addresses: kubbysholly2007@yahoo.com (J. O. Kuboye), qomotolaniq@gmail.com (O. F. Quadri), opeyemielusakin21@gmail.com (O. R. Elusakin)
[4], Olabode [6], Olabode [7], Omar and Suleiman [8], Sagir [9], Sunday [10] amongst others. The accuracy of the methods developed by these researchers are not efficient in terms of error, as this can be improved by the introduction of off grid points within the interval of integration.

Hence this paper addresses the shortcoming of low accuracy found in the existing method mentioned above.

\section{Methodology}

\subsection{Derivation of First Block Method (FBM)}

This section focuses on the derivation of first block method. A power series

$y(x)=\sum_{j=0}^{k+4} a_{j} x^{j}$ 
is considered as an approximate solution to third order ordinary differential equations of the form $y^{\prime \prime \prime}=f\left(x, y(x), y^{\prime}(x), y^{\prime \prime}(x)\right)$, $y\left(x_{0}\right)=y_{0}, y^{\prime}\left(x_{0}\right)=y_{0}^{\prime}, y^{\prime \prime}\left(x_{0}\right)=y_{0}^{\prime \prime}$. Equation (1) is differentiated thrice to give:

$y^{\prime \prime \prime}(x)=\sum_{j=0}^{k+4} j(j-1)(j-2) a_{j} x^{j-3}$

where step number $\mathrm{k}=4$. Interpolating (1) at $x=x_{n+i}, i=1,2$, $\frac{9}{4}$ and collocating its highest derivative at $x=x_{n+m}, i=0(1) 4$ and $\frac{9}{4}$, the resulting equations produce a system of non-linear equations of the form

$$
\begin{aligned}
& \sum_{j=0}^{k+4} a_{j} x_{n+i}^{j}=y_{n+i} \\
& \sum_{j=3}^{k+4} j(j-1)(j-2) a_{j} x_{n+m}^{j-3}=f_{n+m}
\end{aligned}
$$

Solving for the unknowns constants $a_{j}, j=0(1) 8$ in (3) using Gaussian elimination approach and substituting into (1) to give a continuous implicit scheme of the form

$\sum_{j=1}^{k-2} \alpha_{j(t)} y_{n+j}+\alpha_{\frac{9}{4}}(t) y_{n+\frac{9}{4}}=h^{3}\left[\sum_{j=0}^{k} \beta_{j}(t) f_{n+j}+\gamma_{\frac{9}{4}(t)} f_{n+\frac{9}{4}}\right]$

where $t=\frac{x-x_{n+k-1}}{h}$

$\left[\begin{array}{l}\alpha_{1} \\ \alpha_{2} \\ \alpha_{\frac{9}{4}}\end{array}\right]=\left[\begin{array}{ccc}\frac{3}{5} & \frac{7}{5} & \frac{4}{5} \\ -6 & -11 & -4 \\ \frac{32}{5} & \frac{48}{5} & \frac{16}{5}\end{array}\right]\left[\begin{array}{l}t^{0} \\ t^{1} \\ t^{2}\end{array}\right]$

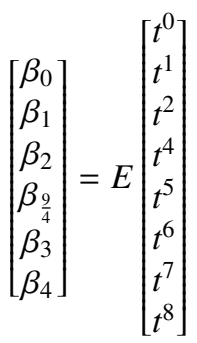

where $E$ has been defined in Appendix B. Equation (4) is differentiated twice and the coefficients are as follow:

$\left[\begin{array}{c}\alpha_{1}^{\prime} \\ \alpha_{2}^{\prime} \\ \alpha_{\frac{9}{4}}^{\prime}\end{array}\right]=\left[\begin{array}{cc}5 & \frac{8}{7} \\ -11 & -8 \\ \frac{48}{5} & \frac{32}{5}\end{array}\right]\left[\begin{array}{l}t^{0} \\ t^{1}\end{array}\right]$

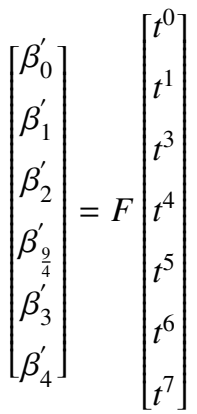

where $F$ has been defined in Appendix B.

$\left[\begin{array}{l}\alpha_{1}^{\prime \prime} \\ \alpha_{2}^{\prime \prime} \\ \alpha_{\frac{9}{4}}^{\prime \prime}\end{array}\right]=\left[\begin{array}{c}\frac{8}{5} \\ -8 \\ \frac{32}{5}\end{array}\right]\left[t^{0}\right]$

$\left[\begin{array}{c}\beta_{0}^{\prime \prime} \\ \beta_{1}^{\prime \prime} \\ \beta_{2}^{\prime \prime} \\ \beta_{9}^{\prime \prime} \\ \beta_{3}^{\prime \prime} \\ \beta_{4}^{\prime \prime}\end{array}\right]=G\left[\begin{array}{l}t^{0} \\ t^{2} \\ t^{3} \\ t^{4} \\ t^{5} \\ t^{6}\end{array}\right]$

and

$G=\left[\begin{array}{cccccc}\frac{-573440}{185794560} & \frac{-1892352}{185794560} & \frac{430080}{185794560} & \frac{3153920}{185794560} & \frac{2580480}{185794560} & \frac{-585111}{185794560} \\ \frac{114688}{5160960} & \frac{516096}{5160960} & \frac{215040}{5160960} & \frac{-860160}{5160960} & \frac{-774144}{5160960} & \frac{262947}{5160960} \\ \frac{-573440}{3440640} & \frac{-3268608}{3440640} & \frac{-3440640}{3440640} & \frac{6021120}{3440640} & \frac{7741440}{3440640} & \frac{496249}{3440640} \\ \frac{114688}{635040} & \frac{688128}{635040} & \frac{860160}{635040} & \frac{-1146880}{635040} & \frac{-2064384}{635040} & \frac{487059}{635040} \\ \frac{-573440}{15482880} & \frac{-3956736}{15482880} & \frac{-7526400}{15482880} & \frac{1433600}{15482880} & \frac{16773120}{15482880} & \frac{4595169}{15482880} \\ \frac{573440}{144506880} & \frac{4644864}{144506880} & \frac{13332480}{144506880} & \frac{16343040}{144506880} & \frac{7741440}{144506880} & \frac{-837513}{144506880}\end{array}\right]$

The discrete schemes and its derivatives which are generated from (4) are represented in a matrix to form a block

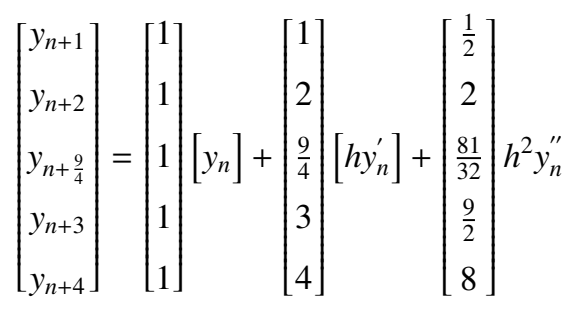

$$
+h^{3}\left[\begin{array}{ccccc}
0 & 0 & 0 & 0 & \frac{4229}{45360} \\
0 & 0 & 0 & 0 & \frac{2699}{5670} \\
0 & 0 & 0 & 0 & \frac{45392643}{73400320} \\
0 & 0 & 0 & 0 & \frac{81}{70} \\
0 & 0 & 0 & 0 & \frac{6056}{2835}
\end{array}\right]\left[\begin{array}{c}
f_{n-4} \\
f_{n-3} \\
f_{n-2} \\
f_{n-1} \\
f_{n}
\end{array}\right]
$$

$$
+h^{3}\left[\begin{array}{ccccc}
\frac{271}{1680} & \frac{-19}{40} & \frac{8896}{19845} & \frac{-1003}{15120} & \frac{63}{11760} \\
\frac{148}{105} & \frac{-64}{21} & \frac{8192}{2835} & \frac{-404}{945} & \frac{1}{30} \\
\frac{5137263}{2621440} & \frac{-144020511}{36700160} & \frac{943569}{250880} & \frac{-10281087}{18350080} & \frac{22497669}{22497669} \\
\frac{2349}{560} & \frac{-243}{35} & \frac{1728}{245} & \frac{-117}{112} & \frac{81}{980} \\
\frac{128}{15} & \frac{-416}{35} & \frac{262144}{19845} & \frac{-1408}{945} & \frac{8}{49}
\end{array}\right]\left[\begin{array}{c}
f_{n+1} \\
f_{n+2} \\
f_{n+1} \\
f_{n+3} \\
f_{n+4}
\end{array}\right]
$$

\subsection{Derivation of Second Block Method(SBM)}

Equation (1) is interpolated at $x=x_{n+j}, j=1,2$ and $\frac{5}{2}$. Equation(2) is collocated at $x=x_{n+j}, j=0(1) 4$ and $\frac{5}{2}$. The 
same step employed in deriving the first block method (FBM) are also used and this gives the second block of the form

$$
\begin{aligned}
& {\left[\begin{array}{l}
y_{n+1} \\
y_{n+2} \\
y_{n+\frac{5}{2}} \\
y_{n+3} \\
y_{n+4}
\end{array}\right]=\left[\begin{array}{l}
1 \\
1 \\
1 \\
1 \\
1
\end{array}\right]\left[y_{n}\right]+\left[\begin{array}{l}
1 \\
2 \\
\frac{5}{2} \\
3 \\
4
\end{array}\right]\left[h y_{n}^{\prime}\right]+\left[\begin{array}{c}
\frac{1}{2} \\
2 \\
\frac{25}{8} \\
\frac{9}{2} \\
8
\end{array}\right] h^{2} y_{n}^{\prime \prime}} \\
& +h^{3}\left[\begin{array}{ccccc}
0 & 0 & 0 & 0 & \frac{379}{4032} \\
0 & 0 & 0 & 0 & \frac{101}{210} \\
0 & 0 & 0 & 0 & \frac{116125}{147456} \\
0 & 0 & 0 & 0 & \frac{2619}{2240} \\
0 & 0 & 0 & 0 & \frac{136}{63}
\end{array}\right]\left[\begin{array}{c}
f_{n-4} \\
f_{n-3} \\
f_{n-2} \\
f_{n-1} \\
f_{n}
\end{array}\right] \\
& +h^{3}\left[\begin{array}{ccccc}
\frac{115}{756} & \frac{-901}{3360} & \frac{278}{945} & \frac{-283}{2520} & \frac{59}{8640} \\
\frac{1276}{945} & \frac{-12}{7} & \frac{256}{135} & \frac{-76}{105} & \frac{83}{1890} \\
\frac{1943125}{774144} & \frac{-460625}{172032} & \frac{75125}{24192} & \frac{-308125}{258048} & \frac{225625}{3096576} \\
\frac{81}{20} & \frac{-4131}{1120} & \frac{162}{35} & \frac{-99}{56} & \frac{243}{2240} \\
\frac{7808}{945} & \frac{-608}{105} & \frac{8192}{945} & \frac{-128}{45} & \frac{40}{189}
\end{array}\right]\left[\begin{array}{l}
f_{n+1} \\
f_{n+2} \\
f_{n+\frac{5}{2}} \\
f_{n+3} \\
f_{n+4}
\end{array}\right]
\end{aligned}
$$

\section{Analysis of the Method}

\subsection{Order of the method}

The method proposed by Lambert [5] is applied in finding the order of the methods (11) and (12) where y-and f-functions are expanded in Taylor series and comparing the coefficients of $\mathrm{h}$, this gives the methods to be of order $[6,6,6,6,6]^{T}$

\subsection{Zero Stability}

The Continuous implicit scheme (4) is zero-stable if the roots

$r_{n}=1,2, \ldots, N$ of the first characteristic polynomial defined by $p(r)=\operatorname{det}\left[A^{\prime}-B^{\prime}\right]$ satisfy $\left|r_{n}\right| \leq 1$ and $|r|=1$

where

$A^{\prime}=\left[\begin{array}{lllll}1 & 0 & 0 & 0 & 0 \\ 0 & 1 & 0 & 0 & 0 \\ 0 & 0 & 1 & 0 & 0 \\ 0 & 0 & 0 & 1 & 0 \\ 0 & 0 & 0 & 0 & 1\end{array}\right]$ and $B^{\prime}=\left[\begin{array}{ccccc}0 & 0 & 0 & 0 & 1 \\ 0 & 0 & 0 & 0 & 1 \\ 0 & 0 & 0 & 0 & 1 \\ 0 & 0 & 0 & 0 & 1 \\ 0 & 0 & 0 & 0 & 1\end{array}\right]$

Applying the condition above the methods are found to be zero stable.

\subsection{Convergence}

According to Henrici [3], equation(4) converges if it is zerostable and consistent. Therefore, since the order of the methods are greater than one and also satisfies other conditions of zero stability. Hence, the new methods (11) and (12) converged.

\section{Application of the method}

The following problems are considered in order to examine the accuracy of the new block methods.

Problem 1: Consider the initial value problem(I.V.P)

$y^{\prime \prime \prime}=3 \sin x, y(0)=1, y^{\prime}(0)=0, y^{\prime \prime}(0)=-2, h=0.1$

Theoretical solution $y(x)=3 \cos x+\frac{x^{2}}{2}-2$

Problem 2: Consider the I.V.P

$y^{\prime \prime \prime}-y^{\prime \prime}+y^{\prime}-y=0 y(0)=1 y^{\prime}(0)=0 y^{\prime \prime}(0)=-1 h=0.01$

with exact solution of $y(x)=\cos x$

Problem 3: Consider the initial value problem(I.V.P)

$y^{\prime \prime \prime}=\exp x, y(0)=3, y^{\prime}(0)=1, y^{\prime \prime}(0)=5, h=0.1$

Theoretical solution $y(x)=2+2 x^{2}+\exp x$

The following acronyms are used

- $\mathrm{ES}=$ Exact Solution

- $\mathrm{CS}=$ Computed Solution

- EIFBM=Error in first block method with $s=5 / 2$

- $\mathrm{EISBM}=$ Error in second block method with $\mathrm{s}=9 / 4$

- EI0(2009)=Error in Olabode(2009)

- $\operatorname{EIO}(2013)=$ Error in Olabode(2013)

- $\operatorname{EIS}(2014)=$ Error in Sagir(2014)

- EISU(2018)=Error in J.Sunday (2018)

\section{Discussion of Results}

In Table 1, the results generated from the new developed block methods (EIFBM and EISBM) are compared with EIO(2009) and $\operatorname{EIO}(2013)$. It is clearly shown that the accuracy in terms of error reveals the efficiency of the new derived methods over $\mathrm{EIO}(2009,2013)$ inspite of the high step number $\mathrm{k}$ considered. Furthermore, the results produced from the new numerical models outperform the results in EIS(2014) and EISU(2018) as evident in Table 2. Additionally, the effectiveness of the new developed numerical methods is demonstrated in Table 3 as it compared favorably with $\mathrm{EIO}(2013)$ in terms of error. 
Table 1: Comparing the new method with Olabode(2009, 2013) for solving problem 1

\begin{tabular}{ccccccc}
\hline x-value & $E S$ & $C S$ & $E I F B M$ & $E I S B M$ & $E I O(2009)$ & $E I O(2013)$ \\
\hline 0.1 & 0.990012495834077020 & 0.990012495833478170 & $6.8911543 \mathrm{E}-13$ & $5.9885430 \mathrm{E}-13$ & $3.4077519 \mathrm{E}-11$ & $1.65922 \mathrm{E}-10$ \\
0.2 & 0.960199733523725120 & 0.960199733519903840 & $4.4015902 \mathrm{E}-12$ & $3.8212766 \mathrm{E}-12$ & $1.2372514 \mathrm{E}-10$ & $4.76275 \mathrm{E}-10$ \\
0.3 & 0.911009467376818090 & 0.911009467367234980 & $1.0999868 \mathrm{E}-11$ & $9.5831121 \mathrm{E}-12$ & $1.7681812 \mathrm{E}-10$ & $6.23182 \mathrm{E}-10$ \\
0.4 & 0.843182982008655380 & 0.843182981990707400 & $2.0601632 \mathrm{E}-11$ & $1.7947976 \mathrm{E}-11$ & $4.0865533 \mathrm{E}-10$ & $19.9134 \mathrm{E}-10$ \\
0.5 & 0.757747685671118280 & 0.757747685638492150 & $3.6853520 \mathrm{E}-11$ & $3.2626124 \mathrm{E}-11$ & $3.7111825 \mathrm{E}-10$ & $3.28882 \mathrm{E}-10$ \\
0.6 & 0.656006844729034810 & 0.656006844668665210 & $6.7268413 \mathrm{E}-11$ & $6.0369598 \mathrm{E}-11$ & $7.0964790 \mathrm{E}-10$ & $1.27096 \mathrm{E}-09$ \\
0.7 & 0.539526561853465480 & 0.539526561752478040 & $1.1150603 \mathrm{E}-10$ & $1.0098744 \mathrm{E}-10$ & $7.4653450 \mathrm{E}-10$ & $4.84653 \mathrm{E}-09$ \\
0.8 & 0.410120128041496560 & 0.410120127886882570 & $1.6985002 \mathrm{E}-10$ & $1.5461399 \mathrm{E}-10$ & $1.9585035 \mathrm{E}-09$ & $1.09585 \mathrm{E}-08$ \\
0.9 & 0.269829904811992980 & 0.269829904583073650 & $2.4948449 \mathrm{E}-10$ & $2.2891933 \mathrm{E}-10$ & $3.8880070 \mathrm{E}-09$ & $2.0188 \mathrm{E}-08$ \\
1.0 & 0.120906917604419300 & 0.120906917269670430 & $3.6226498 \mathrm{E}-10$ & $3.3474887 \mathrm{E}-10$ & $6.3955807 \mathrm{E}-09$ & $3.53956 \mathrm{E}-08$ \\
1.1 & -0.034211635723267797 & -0.034211636195096484 & $5.0769700 \mathrm{E}-10$ & $4.7182869 \mathrm{E}-10$ & $9.5232678 \mathrm{E}-09$ & $5.66233 \mathrm{E}-08$ \\
1.2 & -0.192926736569979610 & -0.192926737210326740 & $6.8618927 \mathrm{E}-10$ & $6.4034714 \mathrm{E}-10$ & $1.3169979 \mathrm{E}-08$ & $8.35700 \mathrm{E}-08$ \\
\hline
\end{tabular}

Table 2: Comparing the new method with Sagir(2014)and J.Sunday(2018) for solving problem 2

\begin{tabular}{ccccccc}
\hline X-VALUE & $E S$ & $C S$ & $E I F B M$ & $E I S B M$ & $E I S(2014)$ & $E I S U(2018)$ \\
\hline 0.01 & 0.999950000416665260 & 0.999950000416665370 & $1.1102230 \mathrm{E}-16$ & $0.0000000 \mathrm{E}+00$ & $1.9990 \mathrm{E}-07$ & $1.1102 \mathrm{E}-016$ \\
0.02 & 0.999800006666577760 & 0.999800006666578310 & $5.5511151 \mathrm{E}-16$ & $5.5511151 \mathrm{E}-16$ & $1.9560 \mathrm{E}-07$ & $1.3323 \mathrm{E}-015$ \\
0.03 & 0.999550033748987540 & 0.999550033748996200 & $8.6597396 \mathrm{E}-15$ & $8.7707619 \mathrm{E}-15$ & $1.3651 \mathrm{E}-07$ & $9.6589 \mathrm{E}-015$ \\
0.04 & 0.999200106660977920 & 0.999200106661042750 & $6.4837025 \mathrm{E}-14$ & $6.4614980 \mathrm{E}-14$ & $2.5210 \mathrm{E}-07$ & $3.2974 \mathrm{E}-014$ \\
0.05 & 0.998750260394966280 & 0.998750260395229290 & $2.6301183 \mathrm{E}-14$ & $2.6290081 \mathrm{E}-14$ & $1.3039 \mathrm{E}-06$ & $8.2379 \mathrm{E}-014$ \\
\hline
\end{tabular}

Table 3: Comparing the new method with OLABODE(2013) for solving problem 3

\begin{tabular}{cccccc}
\hline X-VALUE & $E S$ & $C S$ & $E I F B M$ & EISBM & EIO $(2013)$ \\
\hline 0.1 & 3.125170918075647700 & 3.125170918077170500 & $1.5227819 \mathrm{e}-12$ & $1.3447021 \mathrm{e}-12$ & $9.24352 \mathrm{E}-10$ \\
0.2 & 3.301402758160169700 & 3.301402758169862000 & $9.6922470 \mathrm{e}-12$ & $8.5487173 \mathrm{e}-12$ & $18.3983 \mathrm{E}-10$ \\
0.3 & 3.529858807576003300 & 3.529858807600271000 & $2.4267699 \mathrm{e}-11$ & $2.1475266 \mathrm{e}-11$ & $24.2400 \mathrm{E}-10$ \\
0.4 & 3.811824697641270600 & 3.811824697686721800 & $4.5451198 \mathrm{e}-11$ & $4.0219827 \mathrm{e}-11$ & $53.5873 \mathrm{E}-10$ \\
0.5 & 4.148721270700128200 & 4.148721270778516200 & $7.8387963 \mathrm{e}-11$ & $7.0274453 \mathrm{e}-11$ & $7.00128 \mathrm{E}-10$ \\
0.6 & 4.542118800390509700 & 4.542118800522103200 & $1.3159340 \mathrm{e}-10$ & $1.1942358 \mathrm{e}-10$ & $3.90509 \mathrm{E}-10$ \\
0.7 & 4.993752707470477500 & 4.993752707675188400 & $2.0471091 \mathrm{e}-10$ & $1.8746782 \mathrm{e}-10$ & $6.52952 \mathrm{E}-09$ \\
0.8 & 5.505540928492468600 & 5.505540928790510200 & $2.9804159 \mathrm{e}-10$ & $2.7454572 \mathrm{e}-10$ & $2.15075 \mathrm{E}-08$ \\
0.9 & 6.07960311156950000 & 6.079603111576208400 & $4.1925841 \mathrm{e}-10$ & $3.8885162 \mathrm{e}-10$ & $3.88430 \mathrm{E}-08$ \\
1.0 & 6.718281828459045500 & 6.718281829040118500 & $5.8107297 \mathrm{e}-10$ & $5.4199667 \mathrm{e}-10$ & $6.15410 \mathrm{E}-08$ \\
\hline
\end{tabular}




\section{Conclusion}

The methods are derived via multistep collocation technique which is used to develop the block methods. The methods are zero stable, consistent, converged and of order six. The accuracy of the methods is better when compared with existing methods in terms of error as displayed in Tables 1,2 and 3. Hence FBM and SBM are reliable and efficient numerical models for solving third order initial value problems of ordinary differential equations.

\section{References}

[1] D. A. Awoyemi, "Class of continuous methods for general second order initial value problems in ordinary differential equations", International Journal of Computer Mathematics 72 (2009) 29.

[2] S. O. Fatunla, Numerical methods for initial value problems in ordinary differential equations, Academic press Inc. Harcourt Brace Jovanovich Publishers, New York (1988)

[3] P. Henrici, "Discrete variable methods in Ordinary Differential Equations", New York, NY; Wiley 571.91 (1962) 419.

[4] J. O. Kuboye \& Z. Omar, "Numerical solution of third order ordinary differential equations using a seven-step block method", International Journal of Mathematical Analysis 9 (2015) 743.

[5] J. D. Lambert, "Computational Method in Ordinary Differential Equation", John Wiley and Sons, Inc 571.91 (1973) 288.

[6] B. T. Olabode, "A six-step scheme for the solution of fourth order ordinary differential equation", The Pacific Journal of Science and Technology 10 (2009) 143.

[7] B. T. Olabode, "Block multistep method for third order ordinary differential equations", FUTA Journal of Research in Sciences 2 (2013) 194.

[8] Z. Omar \& M. Suleiman, "Solving higher order odes directly using parallel 2-point explicit block method", Matematika. Pengintegrasian Teknologi Dalam Sains Matematik. Universiti Sains Malaysia 21 (2005) 15.

[9] A. M. Sagir, "On the Approximate Solution of Continuous Coefficients for Solving Third Order Ordinary Differential Equations", International Journal of Mathematical, Computational, Physical, Electrical and Computer Engineering 8 (2018) 67.

[10] J. Sunday, "On the Oscillation Criteria and Computation of Third Order Oscillatory Differential Equations", Communications in Mathematics and Applications 9 (2018) 615.

\section{Appendix A}

$$
\begin{aligned}
& a_{0}=\frac{(x n+h s) \times(2 h+x n)}{h^{2}(s-1)} y_{n+1}-\frac{(x n+h s) \times(h+x n)}{h^{2}(s-2)} y_{n+2} \\
& +\frac{2 h^{2}+3 h x n+x n^{2}}{h^{2}\left(s^{2}-3 s+2\right)} y_{n+s} \\
& -\frac{2 h^{2}+3 h x n+x n^{2}}{40320 h^{5} s}\left(3 h^{6} s^{6}-51 h^{6} s^{5}\right. \\
& +331 h^{6} s^{4}-1005 h^{6} s^{3}+1363 h^{6} s^{2}-425 h^{6} s+1228 h^{5} s x n \\
& -425 h^{5} x n+1420 h^{4} s x n^{2}-135 h^{4} x n^{2}+616 h^{3} s x n^{3}+415 h^{3} x n^{3} \\
& \left.+116 h^{2} s x n^{4}+285 h^{2} x n^{4}+8 h s x n^{5}+65 h x n^{5}+5 x n^{6}\right) f_{n} \\
& +\frac{2 h^{2}+3 h x n+x n^{2}}{10080 h^{5}(s-1)}\left(3 h^{6} s^{6}-45 h^{6} s^{5}\right. \\
& +223 h^{6} s^{4}-249 h^{6} s^{3}-1193 h^{6} s^{2}+1161 h^{6} s-1586 h^{5} s x n \\
& +1161 h^{5} x n+258 h^{4} s x n^{2}-393 h^{4} x n^{2}+406 h^{3} s x n^{3}+9 h^{3} x n^{3}
\end{aligned}
$$

$$
\begin{aligned}
& \left.+102 h^{2} s x n^{4}+183 h^{2} x n^{4}+8 h s x n^{5}+57 h x n^{5}+5 x n^{6}\right) f_{n+1} \\
& -\frac{2 h^{2}+3 h x n+x n^{2}}{6720 h^{5}(s-2)}\left(3 h^{6} s^{6}-39 h^{6} s^{5}\right. \\
& +143 h^{6} s^{4}+3 h^{6} s^{3}-277 h^{6} s^{2}+31 h^{6} s-228 h^{5} s x n+31 h^{5} x n \\
& -92 h^{4} s x n^{2}+49 h^{4} x n^{2}+252 h^{3} s x n^{3}-89 h^{3} x n^{3}+88 h^{2} s x n^{4} \\
& \left.+109 h^{2} x n^{4}+8 h s x n^{5}+49 h x n^{5}+5 x n^{6}\right) f_{n+2} \\
& +\frac{2 h^{2}+3 h x n+x n^{2}}{336 h^{5} s\left(s^{4}-10 s^{3}+35 s^{2}-50 s+24\right)}\left(13 h^{6} s^{5}\right. \\
& -h^{6} s^{6}-57 h^{6} s^{4}+83 h^{6} s^{3}+27 h^{6} s^{2}-85 h^{6} s-85 h^{5} x n \\
& \left.-27 h^{4} x n^{2}+83 h^{3} x n^{3}+57 h^{2} x n^{4}+13 h x n^{5}+x n^{6}\right) f_{n+s} \\
& +\frac{2 h^{2}+3 h x n+x n^{2}}{10080 h^{5}(s-3)}\left(3 h^{6} s^{6}-33 h^{6} s^{5}\right. \\
& +91 h^{6} s^{4}+3 h^{6} s^{3}-173 h^{6} s^{2}+49 h^{6} s-158 h^{5} s x n+49 h^{5} x n \\
& -50 h^{4} s x n^{2}+15 h^{4} x n^{2}+154 h^{3} s x n^{3}-47 h^{3} x n^{3}+74 h^{2} s x n^{4} \\
& \left.+63 h^{2} x n^{4}+8 h s x n^{5}+41 h x n^{5}+5 x n^{6}\right) f_{n+3} \\
& -\frac{2 h^{2}+3 h x n+x n^{2}}{40320 h^{5}(s-4)}\left(3 h^{6} s^{6}-27 h^{6} s^{5}\right. \\
& +67 h^{6} s^{4}+3 h^{6} s^{3}-125 h^{6} s^{2}+39 h^{6} s-116 h^{5} s x n+39 h^{5} x n \\
& -36 * h^{4} s x n^{2}+9 h^{4} x n^{2}+112 h^{3} s x n^{3}-33 h^{3} x n^{3}+60 h^{2} s x n^{4} \\
& \left.+45 h^{2} x n^{4}+8 h s x n^{5}+33 h x n^{5}+5 x n^{6}\right) f_{n+4}
\end{aligned}
$$$$
a_{1}=-\frac{2 h+2 x n+h s}{h^{2}(s-1)} y_{n+1}+\frac{h+2 x n+h s}{\left(h^{2}(s-2)\right.} y_{n+2}
$$$$
-\frac{3 h+2 x n}{h^{2}(s 2-3 s+2)} y_{n+s}
$$$$
+\frac{1}{40320 h^{5} s}\left(9 h^{7} s^{6}-153 h^{7} s^{5}+993 h^{7} s^{4}-3015 h^{7} s^{3}\right.
$$$$
+4089 h^{7} s^{2}+1181 h^{7} s-850 h^{7}+6 h^{6} s^{6} x n-102 h^{6} s^{5} x n
$$$$
+662 h^{6} s^{4} x n-2010 h^{6} s^{3} x n+2726 h^{6} s^{2} x n+12198 h^{6} s x n
$$$$
-3090 h^{6} x n+20160 h^{5} s x n^{2}+14000 h^{4} s x n^{3}+6720 h^{4} x n^{3}
$$$$
+4900 h^{3} s x n^{4}+7000 h^{3} x n^{4}+840 h^{2} s x n^{5}+2940 h^{2} x n^{5}+56 h s x n^{6}
$$$$
\left.+560 h x n^{6}+40 x n^{7}\right) f_{n}
$$$$
-\frac{1}{10080 h^{5}(s-1)}\left(9 h^{7} s^{6}-135 h^{7} s^{5}+669 h^{7} s^{4}-747 h^{7} s^{3}\right.
$$$$
-3579 h^{7} s^{2}+311 h^{7} s+2322 h^{7}+6 h^{6} s^{6} x n-90 h^{6} s^{5} x n
$$$$
+446 h^{6} s^{4} x n-498 h^{6} s^{3} x n-2386 h^{6} s^{2} x n-6162 h^{6} s x n+5394 h^{6} x n
$$$$
+6720 h^{4} s x n^{3}+3640 h^{3} s x n^{4}+3360 h^{3} x n^{4}+756 h^{2} s x n^{5}
$$$$
\left.+2184 h^{2} x n^{5}+56 h s x n^{6}+504 h x n^{6}+40 x n^{7}\right) f_{n+1}
$$$$
+\frac{1}{6720 h^{5}(s-2)}\left(9 h^{7} s^{6}-117 h^{7} s^{5}+429 h^{7} s^{4}+9 h^{7} s^{3}\right.
$$$$
-831 h^{7} s^{2}-363 h^{7} s+62 h^{7}+6 h^{6} s^{6} x n-78 h^{6} s^{5} x n
$$$$
+286 h^{6} s^{4} x n+6 h^{6} s^{3} x n-554 h^{6} s^{2} x n-1674 h^{6} s x n+382 h^{6} x n
$$$$
+3360 h^{4} s x n^{3}+2660 h^{3} s x n^{4}+1680 h^{3} x n^{4}+672 h^{2} s x n^{5}
$$$$
\left.+1596 h^{2} x n^{5}+56 h s x n^{6}+448 h x n^{6}+40 x n^{7}\right) f_{n+2}
$$$$
-\frac{1}{336 h^{5} s\left(s^{4}-10 s^{3}+35 s^{2}-50 s+24\right)}\left(39 h^{7} s^{5}-3 h^{7} s^{6}\right.
$$ 
$-171 h^{7} s^{4}+249 h^{7} s^{3}+81 h^{7} s^{2}-255 h^{7} s-170 h^{7}-2 h^{6} s^{6} x n$

$+26 h^{6} s^{5} x n-114 h^{6} s^{4} x n+166 h^{6} s^{3} x n+54 h^{6} s^{2} x n-170 h^{6} s x n$

$-618 h^{6} x n+1344 h^{4} x n^{3}+1400 h^{3} x n^{4}+588 h^{2} x n^{5}$

$\left.+112 h x n^{6}+8 x n^{7}\right) f_{n+s}$

$-\frac{1}{10080 h^{5}(s-3)}\left(9 h^{7} s^{6}-99 h^{7} s^{5}+273 h^{7} s^{4}+9 h^{7} s^{3}\right.$

$-519 h^{7} s^{2}-169 h^{7} s+98 h^{7}+6 h^{6} s^{6} x n-66 h^{6} s^{5} x n$

$+182 h^{6} s^{4} x n+6 h^{6} s^{3} x n-346 h^{6} s^{2} x n-1050 h^{6} s x n+354 h^{6} x n$

$+2240 h^{4} s x n^{3}+1960 h^{3} s x n^{4}+1120 h^{3} x n^{4}+588 h^{2} s x n^{5}$

$\left.+1176 h^{2} x n^{5}+56 h s x n^{6}+392 h x n^{6}+40 x n 7\right) f_{n+3}$

$+\frac{1}{40320 h^{5}(s-4)}\left(9 h^{7} s^{6}-81 h^{7} s^{5}+201 h^{7} s^{4}+9 h^{7} s^{3}\right.$

$-375 h^{7} s^{2}-115 h^{7} s+78 h^{7}+6 h^{6} s^{6} x n-54 h^{6} s^{5} x n+134 h^{6} s^{4} x n$

$+6 h^{6} s^{3} x n-250 h^{6} s^{2} x n-762 h^{6} s x n+270 h^{6} x n+1680 h^{4} s x n^{3}$

$+1540 h^{3} s x n^{4}+840 h^{3} x n^{4}+504 h^{2} s x n^{5}+924 h^{2} x n^{5}$

$\left.+56 h s x n^{6}+336 h x n^{6}+40 x n^{7}\right) f_{n+4}$

$a_{2}=\frac{1}{h^{2}(s-1)} y_{n+1}-\frac{1}{h^{2}(s-2)} y_{n+2}+\frac{1}{h^{2}\left(s^{2}-3 s+2\right)} y_{n+s}$

$-\frac{1}{40320 h^{5} s}\left(3 h^{6} s^{6}-51 h^{6} s^{5}+331 h^{6} s^{4}-1005 h^{6} s^{3}\right.$

$+1363 h^{6} s^{2}+6099 h^{6} s-1545 h^{6}+20160 h^{5} s x n+21000 h^{4} s x n^{2}$

$+10080 h^{4} x n^{2}+9800 h^{3} s x n^{3}+14000 h^{3} x n^{3}+2100 h^{2} s x n^{4}$

$\left.+7350 h^{2} x n^{4}+168 h s x n^{5}+1680 h x n^{5}+140 x n^{6}\right) f_{n}$

$+\frac{1}{10080 h^{5}(s-1)}\left(3 h^{6} s^{6}-45 h^{6} s^{5}+223 h^{6} s^{4}-249 h^{6} s^{3}\right.$

$-1193 h^{6} s^{2}-3081 h^{6} s+2697 h^{6}+10080 h^{4} s x n^{2}+7280 h^{3} s x n^{3}$

$\left.+6720 h^{3} x n^{3}+1890 h^{2} s x n^{4}+5460 h^{2} x n^{4}+168 h s x n^{5}+1512 h x n^{5}+140 x n^{6}\right) f_{n+1}$

$-\frac{1}{6720 h^{5}(s-2)}\left(3 h^{6} s^{6}-39 h^{6} s^{5}+143 h^{6} s^{4}+3 h^{6} s^{3}\right.$

$-277 h^{6} s^{2}-837 h^{6} s+191 h^{6}+5040 h^{4} s x n^{2}+5320 h^{3} s x n^{3}$

$\left.+3360 h^{3} x n^{3}+1680 h^{2} s x n^{4}+3990 h^{2} x n^{4}+168 h s x n^{5}+1344 h x n^{5}+140 x n^{6}\right) f_{n+2}$

$+\frac{1}{336 h^{5} s\left(s^{4}-10 s^{3}+35 s^{2}-50 s+24\right)}\left(13 h^{6} s^{5}-h^{6} s^{6}-57 h^{6} s^{4}+83 h^{6} s^{3}\right.$

$\left.+27 h^{6} s^{2}-85 h^{6} s-309 h^{6}+2016 h^{4} x n^{2}+2800 h^{3} x n^{3}+1470 h^{2} x n^{4}+336 h x n^{5}+28 x n^{6}\right) f_{n+s}$

$+\frac{1}{10080 h^{5}(s-3)}\left(3 h^{6} s^{6}-33 h^{6} s^{5}+91 h^{6} s^{4}+3 h^{6} s^{3}\right.$

$-173 h^{6} s^{2}-525 h^{6} s+177 h^{6}+3360 h^{4} s x n^{2}+3920 h^{3} s x n^{3}$

$\left.+2240 h^{3} x n^{3}+1470 h^{2} s x n^{4}+2940 h^{2} x n^{4}+168 h s x n^{5}+1176 h x n^{5}+140 x n^{6}\right) f_{n+3}$

$-\frac{1}{40320 h^{5}(s-4)}\left(3 h^{6} s^{6}-27 h^{6} s^{5}+67 h^{6} s^{4}+3 h^{6} s^{3}\right.$

$-125 h^{6} s^{2}-381 h^{6} s+135 h^{6}+2520 h^{4} s x n^{2}+3080 h^{3} s x n^{3}$

$\left.+1680 h^{3} x n^{3}+1260 h^{2} s x n^{4}+2310 h^{2} x n^{4}+168 h s x n^{5}+1008 h x n^{5}+140 x n^{6}\right) f_{n+4}$

$a_{3}=\frac{x n+h s}{144 h^{5} s}\left(24 h^{4}+50 h^{3} x n+35 h^{2} x n^{2}+10 h x n^{3}+x n^{4}\right) f_{n}$ 


$$
\begin{aligned}
& -\frac{x n(x n+h s)}{36 h^{5}(s-1)}\left(24 h^{3}+26 h^{2} x n+9 h x n^{2}+x n^{3}\right) f_{n+1} \\
& +\frac{x n(x n+h s)}{24 h^{5}(s-2)}\left(12 h^{3}+19 h^{2} x n+8 h x n^{2}+x n^{3}\right) f_{n+2} \\
& -\frac{x n}{6 h^{5} s\left(s^{4}-10 s^{3}+35 s^{2}-50 s+24\right)}\left(24 h^{4}+50 h^{3} x n+35 h^{2} x n^{2}+10 h x n^{3}+x n^{4}\right) f_{n+s} \\
& -\frac{x n(x n+h s)}{36 h^{5}(s-3)}\left(8 h^{3}+14 h^{2} x n+7 h x n^{2}+x n^{3}\right) f_{n+3} \\
& +\frac{x n(x n+h s)}{144 h^{5}(s-4)}\left(6 h^{3}+11 h^{2} x n+6 h x n^{2}+x n^{3}\right) f_{n+4} \\
& a_{4}=-\frac{1}{576 h^{5} s}\left(50 h^{4} s+40 h x n^{3}+100 h^{3} x n+24 h^{4}+5 x n^{4}+105 h^{2} x n^{2}+4 h s x n^{3}+70 h^{3} s x n+30 h^{2} s x n^{2}\right) f_{n} \\
& +\frac{1}{144 h^{5}(s-1)}\left(24 h^{4} s+36 h x n^{3}+48 h^{3} x n+5 x n^{4}+78 h^{2} x n^{2}+4 h s x n^{3}+52 h^{3} s x n+27 h^{2} s x n^{2}\right) f_{n+1} \\
& -\frac{1}{96 h^{5}(s-2)}\left(12 h^{4} s+32 h x n^{3}+24 h^{3} x n+5 x n^{4}+57 h^{2} x n^{2}+4 h s x n^{3}+38 h^{3} s x n+24 h^{2} s x n^{2}\right) f_{n+2} \\
& +\frac{1}{24 h^{5} s\left(s^{4}-10 s^{3}+35 s^{2}-50 s+24\right)}\left(24 h^{4}+100 h^{3} x n+105 h^{2} x n^{2}+40 h x n^{3}+5 x n^{4}\right) f_{n+s} \\
& \left.+\frac{1}{144 h^{5}(s-3)}\left(8 h^{4} s+28 h x n^{3}+16 h^{3} x n+5 x n^{4}+42 h^{2} x n^{2}+4 h s x n^{3}+28 h^{3} s x n+21 h^{2} s x n^{2}\right)\right) f_{n+3} \\
& -\frac{1}{576 h^{5}(s-4)}\left(6 h^{4} s+24 h x n^{3}+12 h^{3} x n+5 x n^{4}+33 h^{2} x n^{2}+4 h s x n^{3}+22 h^{3} s x n+18 h^{2} s x n^{2}\right) f_{n+4} \\
& a_{5}=\frac{1}{1440 h^{5} s}\left(35 h^{3} s+60 h x n^{2}+105 h^{2} x n+50 h^{3}+10 x n^{3}+6 h s x n^{2}+30 h^{2} s x n\right) f_{n} \\
& -\frac{1}{360 * h^{5} *(s-1)}\left(26 h^{3} s+54 h x n^{2}+78 h^{2} x n+24 h^{3}+10 x n^{3}+6 h s x n^{2}+27 h^{2} s x n\right) f n+1 \\
& \left.+\frac{1}{240 h^{5}(s-2)}\left(19 h^{3} s+48 h x n^{2}+57 h^{2} x n+12 h^{3}+10 x n^{3}+6 h s x n^{2}+24 h^{2} s x n\right)\right) f_{n+2} \\
& -\frac{1}{12 h^{5} s\left(s^{4}-10 s^{3}+35 s^{2}-50 s+24\right)}\left(10 h^{3}+21 h^{2} x n+12 h x n^{2}+2 x n^{3}\right) f_{n+s} \\
& -\frac{1}{360 h^{5}(s-3)}\left(14 h^{3} s+42 h x n^{2}+42 h^{2} x n+8 h^{3}+10 x n^{3}+6 h s x n^{2}+21 h^{2} s x n\right) f_{n+3} \\
& +\frac{1}{1440 h^{5}(s-4)}\left(11 h^{3} s+36 h x n^{2}+33 h^{2} x n+6 h^{3}+10 x n^{3}+6 h s x n^{2}+18 h^{2} s x n\right) f_{n+4} \\
& a_{6}=-\frac{1}{2880 h^{5} s}\left(40 h x n+10 h^{2} s+35 h^{2}+10 x n^{2}+4 h s x n\right) f_{n} \\
& +\frac{1}{720 h^{5}(s-1)}\left(36 h x n+9 h^{2} s+26 h^{2}+10 x n^{2}+4 h s x n\right) f_{n+1} \\
& -\frac{1}{480 h^{5}(s-2)}\left(32 h x n+8 h^{2} s+19 h^{2}+10 x n^{2}+4 h s x n\right) f_{n+2} \\
& +\frac{7 h^{2}+8 h x n+2 x n^{2}}{24 h^{5} s *\left(s^{4}-10 s^{3}+35 s^{2}-50 s+24\right.} f_{n+s} \\
& +\frac{1}{720 h^{5}(s-3)}\left(28 h x n+7 h^{2} s+14 h^{2}+10 x n^{2}+4 h s x n\right) f_{n+3} \\
& -\frac{1}{2880 h^{5}(s-4)}\left(24 h x n+6 h^{2} s+11 h^{2}+10 x n^{2}+4 h s x n\right) f_{n+4} \\
& a_{7}=\frac{10 h+5 x n+h s}{5040 h^{5} s} f_{n}-\frac{9 h+5 x n+h s}{1260 h^{5}(s-1)} f_{n+1}+\frac{8 h+5 x n+h s}{840 h^{5}(s-2)} f_{n+2} \\
& -\frac{2 h+x n}{42 h^{5} s\left(s^{4}-10 s^{3}+35 s^{2}-50 s+24\right)} f_{n+s}-\frac{7 h+5 x n+h s}{1260^{5}(s-3)} f_{n+3}+\frac{6 h+5 x n+h s}{5040 h^{5}(s-4)} f_{n+4} \\
& a_{8}=-\frac{1}{8064 h^{5} s\left(s^{4}-10 s^{3}+35 s^{2}-50 s+24\right)}\left(24 f_{n}-24 f_{n+s}-50 s f_{n}+96 s f_{n+1}\right.
\end{aligned}
$$




$$
\begin{aligned}
& -72 s f_{n+2}+32 s f_{n+3}-6 s f_{n+4}+35 s^{2} f_{n}-10 s^{3} f_{n}-104 s^{2} f_{n+1}+s^{4} f_{n}+36 s^{3} f_{n+1} \\
& +114 s^{2} f_{n+2} \\
& \left.+11 s^{2} f_{n+4}-4 s^{4} f_{n+3}-6 s^{3} f_{n+4}+s^{4} f_{n+4}\right)
\end{aligned}
$$

\section{Appendix B}

$E=\left[\begin{array}{llllllll}\frac{20480}{371589120} & \frac{90112}{371589120} & \frac{28672}{371589120} & \frac{315392}{371589120} & \frac{430080}{371589120} & \frac{585111}{371589120} & \frac{665797}{371589120} & \frac{236334}{371589120} \\ \frac{4096}{10321920} & \frac{24576}{10321920} & \frac{14336}{10321920} & \frac{86016}{10321920} & \frac{129024}{10321920} & \frac{262947}{10321920} & \frac{346473}{10321920} & \frac{132678}{10321920} \\ \frac{-20480}{6881280} & \frac{-155648}{6881280} & \frac{-229376}{6881280} & \frac{602112}{6881280} & \frac{1290240}{6881280} & \frac{496249}{6881280} & \frac{2271083}{6881280} & \frac{1180914}{6881280} \\ \frac{4096}{1270080} & \frac{32768}{1270080} & \frac{57344}{1270080} & \frac{114688}{1270080} & \frac{344064}{1270080} & \frac{487059}{1270080} & \frac{351161}{1270080} & \frac{64806}{1270080} \\ \frac{-20480}{30965760} & \frac{-188416}{30965760} & \frac{-501760}{30965760} & \frac{143360}{30965760} & \frac{2795520}{30965760} & \frac{4595169}{30965760} & \frac{2242979}{30965760} & \frac{490434}{30965760} \\ \frac{20480}{289013760} & \frac{221184}{289013760} & \frac{888832}{289013760} & \frac{1634304}{289013760} & \frac{1290240}{289013760} & \frac{-837513}{3870720} & \frac{-693915}{289013760} & \frac{-200466}{289013760}\end{array}\right]$
$F=\left[\begin{array}{lllllll}\frac{-163840}{371589120} & \frac{-630784}{371589120} & \frac{172032}{371589120} & \frac{1576960}{371589120} & \frac{1720320}{371589120} & \frac{-1170222}{371589120} & \frac{-665797}{371589120} \\ \frac{32768}{10321920} & \frac{172032}{10321920} & \frac{86016}{10321920} & \frac{-430080}{10321920} & \frac{-516096}{10321920} & \frac{525894}{10321920} & \frac{346473}{10321920} \\ \frac{-163840}{6881280} & \frac{-1089536}{6881280} & \frac{-1376256}{6881280} & \frac{3010560}{6881280} & \frac{5160960}{6881280} & \frac{992498}{6881280} & \frac{2271083}{6881280} \\ \frac{32768}{1270080} & \frac{229376}{1270080} & \frac{344064}{1270080} & \frac{-573440}{1270080} & \frac{-3176256}{1270080} & \frac{974118}{1270080} & \frac{351161}{1270080} \\ \frac{-163840}{30965760} & \frac{-1318912}{30965760} & \frac{-3010560}{30965760} & \frac{716800}{30965760} & \frac{11182080}{30965760} & \frac{9190338}{30965760} & \frac{2242979}{30965760} \\ \frac{163840}{289013760} & \frac{1548288}{289013760} & \frac{752645332992}{289013760} & \frac{8171520}{289013760} & \frac{5160960}{289013760} & \frac{-1675026}{289013760} & \frac{-693915}{289013760}\end{array}\right]$

